\title{
SCANNING MICROLENS SHUTTLES FABRICATED USING SILICON-ON-INSULATOR WAFERS
}

Decai Sun, Chingwen Yeh and Michel Rosa

Xerox Palo Alto Research Center, Palo Alto, CA 94304.

Email: mrosa@parc.xerox.com; Fax: 650-812-4105

\begin{abstract}
Scanning microlens shuttles fabricated using silicon-on-insulator (SOI) wafer material are presented in this paper. Refractive polymer microlens arrays are integrated on top of a single crystal silicon shuttle, which is actuated by a set of comb drives fabricated in the wafers' single-crystal-silicon (SCS) device layer. The lens shuttles have been dynamically operated at resonant frequencies ranging from tens of hertz up to $\sim 10 \mathrm{kHz}$. With a maximum displacement of $\sim 40 \mu \mathrm{m}$ demonstrated at a fundamental resonant frequency of $\sim 4.7 \mathrm{kHz}$, with a quality factor (Q) of $\sim 149$. Upon the SCS shuttle(s), a microlens was fabricated using a photoresist reflow technique. The lenses fabricated using this technique have a calculated focal length of $\sim 808 \mu \mathrm{m}$.
\end{abstract}

\section{Introduction}

Technological development and progress in optical MEMS is driving the desire to make low cost miniaturized optical scanners for applications in the areas of display, printing and more recently optical telecommunications. Such devices can be easily integrated with light sources such as edge emitting lasers and vertical cavity surface emitting lasers to form compact laser scanners. Previously a scanning diffractive Fresnel microlens has been demonstrated using a poly-Si surface MEMS fabrication technique [1]. In this paper we present a refractive polymer microlens array integrated on top of a comb-drive actuated SCS shuttle fabricated using SOI. We believe this to be the first demonstration of such a device ever reported.

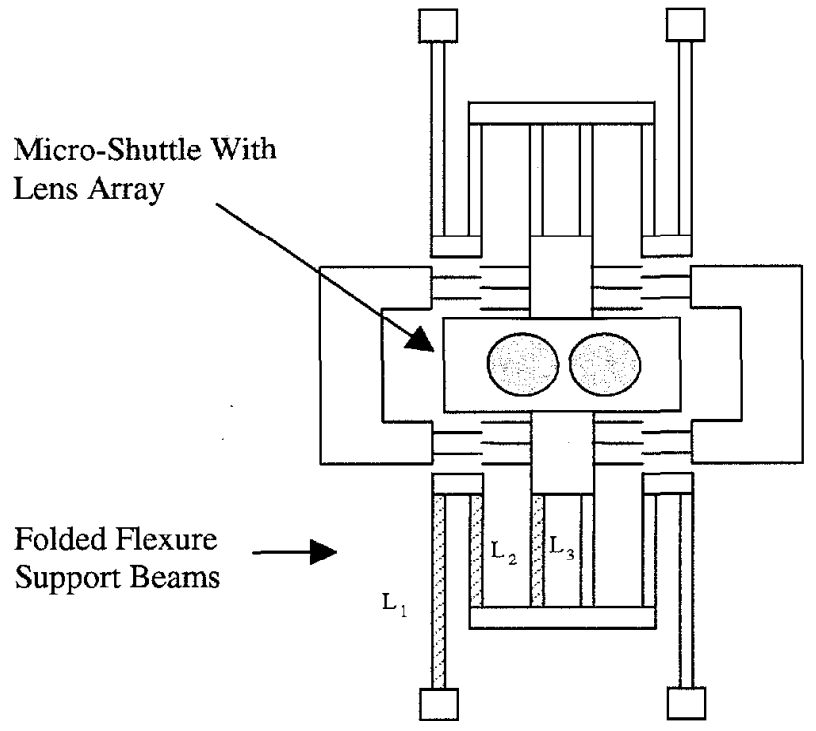

Figure. 1. A comb drive actuated shuttle with a polymer microlens array.

\section{Device Design}

The device shown in Figure 1 has been prototyped for use in laser scanning applications. A SCS stage of $\sim 545 \mu \mathrm{m} \mathrm{x}$ $\sim 300 \mu \mathrm{m}$ suspended by folded springs on either side and actuated by a set of interdigitated comb fingers, is used as the platform for the microlens array. Device actuation is achieved electrostatically by the $100 \mathrm{comb}$ fingers on either side of the suspended stage. The large number of comb fingers is used to generate a large electrostatic force for a relatively low applied voltage, which in combination with a reduced spring constant can more readily facilitate the large displacements required for beam scanning applications [2]. Each of the interdigitated comb fingers is $\sim 55 \mu \mathrm{m}$ long and $\sim 2 \mu \mathrm{m}$ wide, with an interdigitated comb finger gap of 2 $\mu \mathrm{m}$. The schematic of Figure 1, depicts the entire device with $\mathrm{L}_{1}, \mathrm{~L}_{2}$ and $\mathrm{L}_{3}$ representing the lengths of each of the beams in the folded flexure design used to support the lens shuttle. The spring constant calculation used for this support bcam configuration is :

$k_{s}=\left[\frac{\frac{k_{1} * k_{2}}{k_{1}+k_{2}} * k_{3}}{\frac{k_{1} * k_{2}}{k_{1}+k_{2}}+k_{3}}\right] * 4 ;$ where $k_{i}=\frac{12 E I}{L_{i}^{3}}$

The young's modulus used for the calculations was 150Gpa and for devices such as that of Figure 1, the dimensions of beams $\mathrm{L}_{1}, \mathrm{~L}_{2}$ and $\mathrm{L}_{3}$ were $\sim 350 \mu \mathrm{m}, \sim 300 \mu \mathrm{m}$ and $\sim 300 \mu \mathrm{m}$ respectively for a support beam width of $\sim 5 \mu \mathrm{m}$. The resonant frequency of the shuttle is :

$$
f=\frac{1}{2 \pi} \sqrt{\frac{k_{s}}{M}},
$$

where $M$ is the effective mass of the lens shuttle.

The focal length of the refractive polymer lens is,

$$
f=\frac{R}{n-1}
$$

where $\mathrm{n}$ is the refractive index of the polymer, and $\mathrm{R}$ is the radius of curvature of the spherical photoresist lens. The thickness and the diameter of the photoresist lens before reflow determine its radius of curvature and therefore the final focal length. 


\section{Fabrication}

Typically, comb-drive based devices are fabricated using surface micro-machining techniques on deposited polysilicon layers [3], [4]. Previously, it has been found that the use of SOI allows simple device fabrication requiring only one masking step [5]. Such techniques were applied here to reduce the fabrication complexity of the microlens devices. The wafer material used for the fabrication process consisted of 4-inch (100) BESOI (Bond and Etch Back Silicon-On-Insulator) wafers with a device layer thickness of $10 \mu \mathrm{m}$, a buried oxide thickness of $0.5 \mu \mathrm{m}$ and a handle wafer thickness of $\sim 420 \mu \mathrm{m}$. The main processing steps toward the fabrication of the microlens devices tested are illustrated in the process flow of Figure 2.

(1)

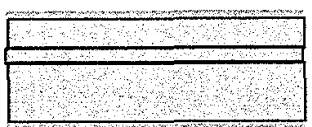

(2)
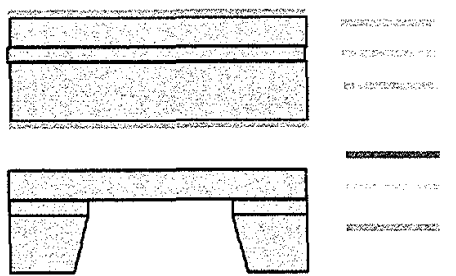

SCS Device Layer Buried Oxide Layer Handle Wafer

Photoresist Tens Aluminum Layer Silicon Nitride Layer

(3)

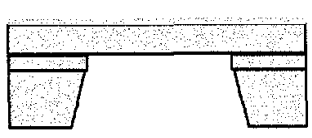

(4)

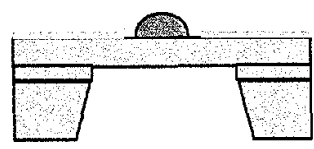

(5)

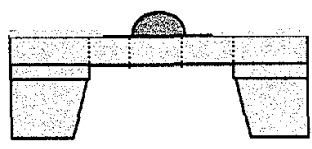

step (3) after the $\operatorname{SiN}_{\mathrm{x}}$ is removed. This layer serves to pattern the electrical contacts for the device. In step (4), photolithography is performed toward the formation of the microlens array. Each lens is measured to be around $5 \mu \mathrm{m}$ in height and $\sim 130 \mu \mathrm{m}$ in diameter after reflow. Reflow of the microlens array after photoresist patterning, takes place at a temperature of $\sim 200^{\circ} \mathrm{C}[6]$. Each lens in the array is spaced at $150 \mu \mathrm{m}$. In a final proccssing step, step (5) involves the Deep Reactive Ion Etch (DRIE) of the $10 \mu \mathrm{m}$ thick SCS device layer. For this step, photoresist is used as a masking layer to protect both the lens array and the $\mathrm{Al}$ regions deposited earlier in the process, while patterning the comb drive device. Using a STS (Surface Technology Systems) Multiplex ICP (Inductively Coupled Plasma), the DRIE process was performed to form and free the shuttle array. Finally the photoresist etch mask is removed in an $\mathrm{O}_{2}$ plasma etcher. The etch rate during this process was $\sim 1.3 \mu \mathrm{m} / \mathrm{min}$.

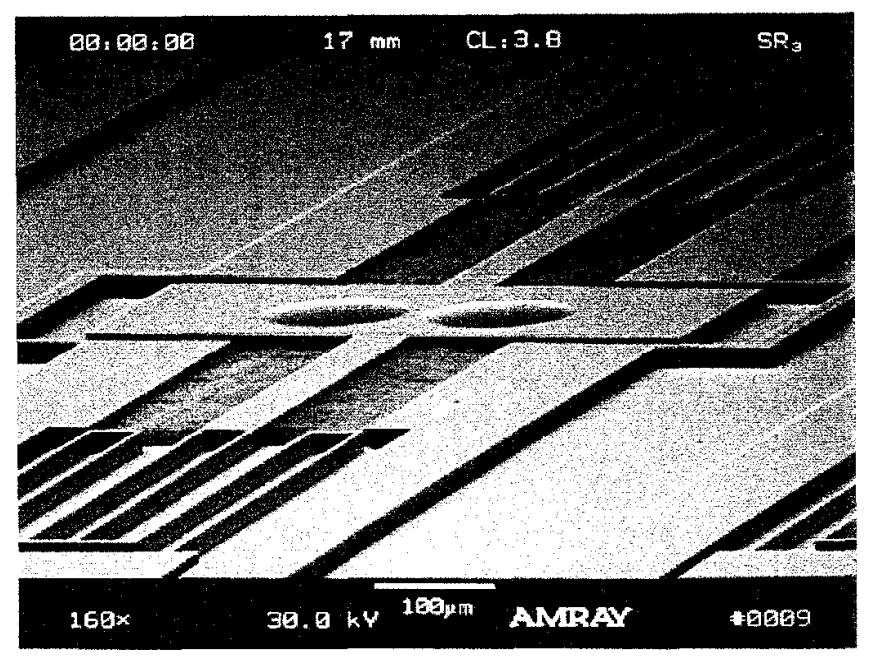

Figure 3. A SCS comb drive-actuated shuttle with a polymer microlens array fabricated on top prior to final release of the shuttle.

Figure 3 shows an SEM photo of a completed device with a set of two microlenses formed on the shuttle mass. Using similar fabrication steps as those previously outlined, the basic device design shown in Figure 3 can be expanded upon to create a super lens shuttle which can be used to provide longer and more seemless scan lines by incorporating a larger number of microlenses. 


\begin{tabular}{|c|c|c|c|c|c|c|c|}
\hline DEVICE & $\begin{array}{c}\mathbf{L}_{1}, \mathbf{L}_{2}, \mathbf{L}_{\mathbf{3}} \\
(\mu \mathbf{m})\end{array}$ & $\begin{array}{c}\text { Beam Width } \\
(\mu \mathrm{m})\end{array}$ & $\begin{array}{c}\mathbf{K s} \\
\text { (Spring Const) }\end{array}$ & $\begin{array}{c}\text { Voltage } \\
(\mathbf{A C})\end{array}$ & $\begin{array}{c}\mathbf{f}_{\mathbf{r}} \\
(\text { Analytical) }\end{array}$ & $\begin{array}{c}\mathbf{f}_{\mathbf{r}} \\
(\text { Experimental) }\end{array}$ & $\begin{array}{c}\text { Displacement } \\
(\text { a.u. })\end{array}$ \\
\hline 2-Lens Array & $540,500,500$ & 4.2 & 1.09096 & 25 & $2268 \mathrm{~Hz}$ & $\sim 4550 \mathrm{~Hz}$ & $\sim 30$ \\
\hline 2-Lens Array & $350,300,300$ & 4.2 & 4.58548 & 25 & $4651 \mathrm{~Hz}$ & $\sim 4700 \mathrm{~Hz}$ & $\sim 30$ \\
\hline
\end{tabular}

Table 1. For the micro-shuttle devices on which an array of two lenses were fabricated, the analytical and experimental characteristics are listed here for each design where the lengths of the support beams $\mathrm{L}_{1}, \mathrm{~L}_{2}$ and $\mathrm{L}_{3}$ are different.

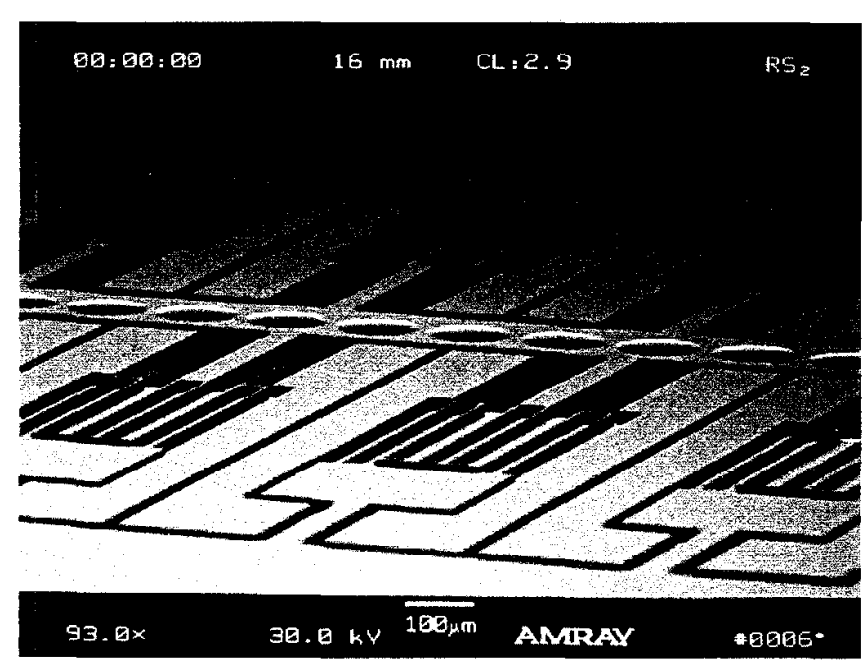

Figure 4. An SEM photograph showing part of a fabricated 13 lensed device.

Figure 4 shows an SEM photo of one such device - a super lens shuttle actuated by multiple parallel comb drive actuators. With a total of 13 microlenses, this is the largest actuated array fabricated in this series of experiments. Other devices which contain an array of 6 microlenses have also been successfully fabricated and tested.

\section{Device Characterization}

After microlens reflow, the thickness of the microlens is measured used a profiler. As shown in Figure 5, the thickness of the spherical lens is $5 \mu \mathrm{m}$. The radius of the lens is about $130 \mu \mathrm{m}$. It is estimated that the focal length of the refractive length is $\sim 808 \mu \mathrm{m}$. As with most comb drive based actuators, testing involved both static and dynamic actuation measurements. A typical device as shown in Figure 3, where L1, L2 and L3 are $350 \mu \mathrm{m}$, $300 \mu \mathrm{m}$ and $300 \mu \mathrm{m}$ respectively, demonstrated a displacement close to $\pm 40 \mu \mathrm{m}$ in resonance with a peak voltage of $25 \mathrm{~V}$. The displacement of the lens shuttle was observed in slow motion with a strobe illumination system. Dynamic testing of this 2-lens device displayed a fundamental resonant frequency of $\sim 4700 \mathrm{~Hz}$. Measurements carried out at and around the fundamental frequency of this device resulted in a $Q$ of $\sim 145$. As such these devices display quality factors well in excess of those readily attainable by typical surface micromachined resonator devices fabricated using polysilicon or other

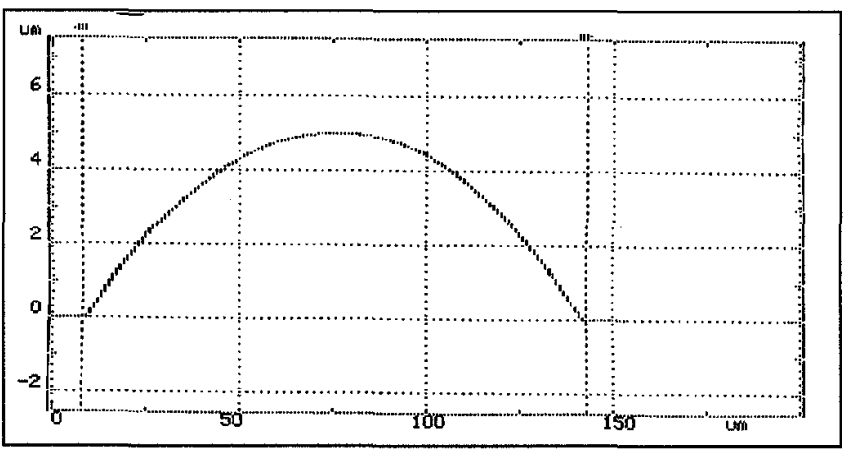

Figure 5. Surface profilometry measurement of a microlens after thermal reflow.

such deposited materials. It is believed that this feature is due in principle to the low air damping and high mechanical quality of the SCS layer in which the comb drive actuator is fabricated. Damping is greatly reduced due to the cavity over which the device is suspended. Formed using the backside $\mathrm{KOH}$ etch described earlier, this cavity enables the device to reach very high $Q$ levels, an artifact that has made the experimental determination of device displacement at and around resonant peaks more complex.

\section{Frequency Response Of Micro-Lens Shuttle}

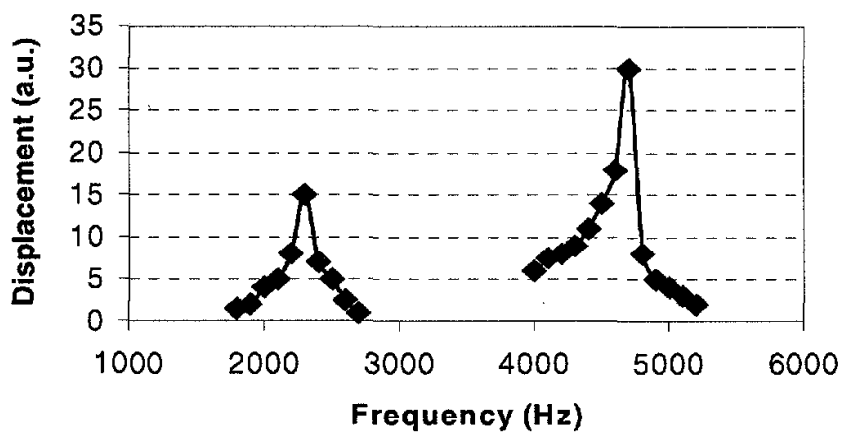

Figure 6. This graph depicts the experimentally determined fundamental frequency peak and its nearest sub-harmonic at $\sim 4.7 \mathrm{kHz}$ and $-1.15 \mathrm{kHz}$, respectively.

Table 1 compares the analytical and experimental fundamental frequencies of both device designs in which for the 2-lens array design, only the support beam length varies. As the data in Table 1 indicates, an increased support beam length and reduced width (due to slight 
DRIE over etch), combine to decrease the spring constant for the device(s), conversely increasing the frequency for each at which the fundamental resonant peak resides. The first of the two devices listed in Table 1 shows a large, almost 2-fold discrepancy between the analytical and cxpcrimental fundamental frequencics. Repeatcd measurement of multiple devices across the wafer resulted in a similar result, leaving the authors unclear as to why this phenomenon has occurred. Figure 6 displays two resonant peaks experimentally derived from a 2-lens array device where $L_{1}, L_{2}$ and $L_{3}$ are $350 \mu \mathrm{m}, 300 \mu \mathrm{m}$ and $300 \mu \mathrm{m}$ respectively. Both displacement curves are measured in absolute units with the devices fundamental frequency having the larger of the two peaks at $\sim 30$ (a.u.) and its sub-harmonic at $\sim 1.150 \mathrm{kHz}$ having a peak of $\sim 15$ (a.u).

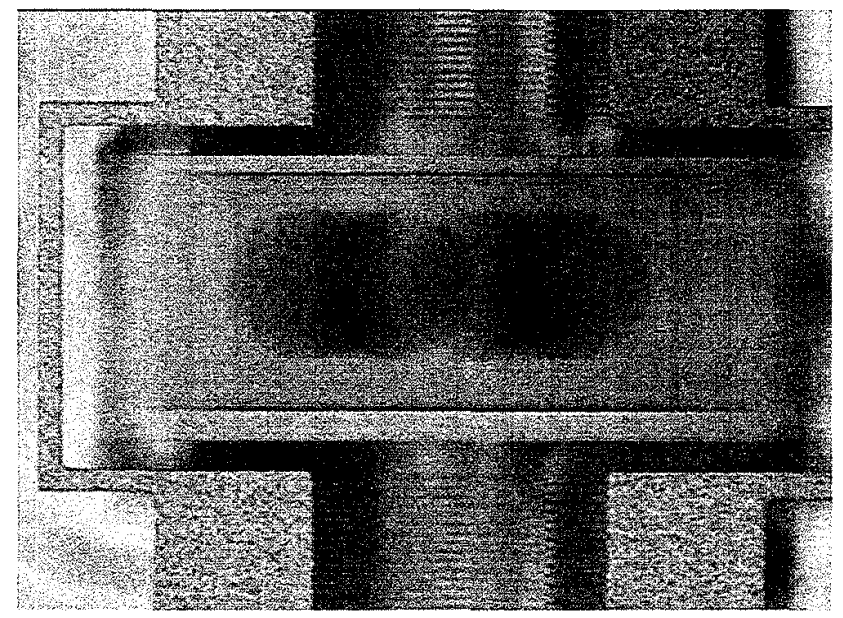

Figure 7. A micro-shuttle with a 2-lens polymer array, at resonance on the probe station.

A typical device with two polymer microlenses is shown at resonance in Figure 7. From the photo in Figure 7, the large displacement achieved while in resonance can easily be seen. This particular shot is taken while at a frequency of $\sim 4700 \mathrm{~Hz}$ and with an applied voltage of around 25 volts (peak). All electrical device testing for these devices was performed using a four-point probe station and both analogue and digital function generators.

\section{Conclusion}

This paper has presented a device concept which would allow the seamless scanning of a laser beam across a given area. Such devices have been fabricated and shown experimentally to provide large displacements for relatively low actuation voltages at high resonant frequencies and $Q$ values. Subsequent to this, the design and fabrication methodologies employed to realize these devices can be expanded upon to allow further device integration which would see these devices perform beam scanning in applications ranging from printing to image scanning.

\section{Acknowledgements}

The authors would like to acknowledge the co-operation of the Stanford Nano-fabrication facility for the use of their DRIE system used to process the wafers in this series of expcriments.

\section{References}

[1]. L. Fan, M. C. Wu, K.D. Choquette, CME2, CLEO'97. [2]. R. Legtenberg, A.W. Groeneveld and $M$. Elwenspoek, "Comb-drive actuators for large displacements", J. Micromech and Microeng, no. 6, pp 320-329, 1996.

[3]. W.C.Tang, T.H.Nguyen, M.W. Judy and R.T. Howe, "Electrostatic-Comb Drive of Lateral Polysilicon Resonators", Sensors And Actuators, A21-A23, pp 328-331, 1990.

[4]. J.J. Sniegowski and E.J. Garcia, "SurfaceMicromachined Gear Trains Driven by an On-Chip Electrostatic Microengine", IEEE Electron Device Letters, pp 366-368. 1996.

[5]. M.A. Rosa, S. Dimitrijev and H.B. Harrison, "Improved Operation of Comb-Drive Actuators through the use of a New Comb Finger Design", J. Inl't Material Systems and Structures, vol. 9, no. 4, pp 283-290, 1998.

[6]. Z.D. Popovic, R.A. Sprague and G.A. Neville Connell, "Technique for monolithic fabrication of microlens arrays", Applied Optics, vol. 27, no. 7, pp $1281-1284,1988$. 